\title{
Hydrodynamic Synchronization of Light Driven Microrotors
}

\author{
R. Di Leonardo, ${ }^{1, *}$ A. Búzás, ${ }^{2}$ L. Kelemen, ${ }^{2}$ G. Vizsnyiczai, ${ }^{2}$ L. Oroszi, ${ }^{2}$ and P. Ormos ${ }^{2}, *$ \\ ${ }^{1}$ IPCF-CNR UOS Roma, Dipartimento di Fisica, Università Sapienza, I-00185 Rome, Italy \\ ${ }^{2}$ Biological Research Center, Hungarian Academy of Sciences, Institute of Biophysics, H-6726 Szeged, Hungary
}

(Received 17 January 2012; revised manuscript received 29 May 2012; published 17 July 2012)

\begin{abstract}
Hydrodynamic synchronization is a fundamental physical phenomenon by which self-sustained oscillators communicate through perturbations in the surrounding fluid and converge to a stable synchronized state. This is an important factor for the emergence of regular and coordinated patterns in the motions of cilia and flagella. When dealing with biological systems, however, it is always hard to disentangle internal signaling mechanisms from external purely physical couplings. We have used the combination of two-photon polymerization and holographic optical trapping to build a mesoscale model composed of chiral propellers rotated by radiation pressure. The two microrotors can be synchronized by hydrodynamic interactions alone although the relative torques have to be finely tuned. Dealing with a micron sized system we treat synchronization as a stochastic phenomenon and show that the phase lag between the two microrotors is distributed according to a stationary Fokker-Planck equation for an overdamped particle over a tilted periodic potential. Synchronized states correspond to minima in this potential whose locations are shown to depend critically on the detailed geometry of the propellers.
\end{abstract}

DOI: 10.1103/PhysRevLett.109.034104

PACS numbers: 05.45.Xt, 05.10.Gg, 47.63.mf, 87.80.Cc

Synchronization is at the basis of a wide variety of fascinating and important phenomena in physics, biology, and engineering. From coupled Josephson junctions [1] to cardiac pacemaker cells [2], the presence of a weak interaction between two or more self-sustained oscillators often leads to the emergence of synchronous patterns [3]. At the micron scale of cells and bacteria, hydrodynamic interactions provide a strong and long-ranged mechanism for coupling [4]. Since synchronization phenomena are known to occur even in the presence of extremely weak and subtle couplings, it is quite natural to expect strong synchronous behavior in such a strongly coupled regime. The presence of a strong coupling, however, is not a sufficient condition for synchronization $[5,6]$, and the role of hydrodynamic interactions for the emergence of synchronous behaviors in flagella [7-9] and cilia [10-13] is still the subject of a lively debate [14]. In the case of waving sheets [5], kinematic reversibility can destroy synchronization when the sheets have reflection symmetry. For the same reason, a collection of rigid rotors, spinning around fixed axes and coupled through hydrodynamic interactions, will appear as the same physical system evolving on a time reversed trajectory when we change sign to all applied torques. Such reversible dynamics cannot give rise to any synchronization behavior that is, by definition, an irreversible process. This symmetry upon torque reversal can be broken by using phase dependent torques [6] or, alternatively, by introducing some degree of mechanical flexibility in the form of internal degrees of freedom with finite stiffness $[15,16]$. In the latter case, when we reverse the sign of applied torques, internal forces will not change their sign and the system will not trace back its history. As a consequence, synchronization in uniformly rotating systems is driven by small deviations from rigid dynamics and amounts to a tiny effect, despite the presence of strong hydrodynamic couplings. In such a situation, synchronization is highly sensitive to a small mismatch in the rotors' free rotational frequencies [16]. An extremely low Reynolds number is an important condition in mesoscopic dynamics, but even more peculiar is the unavoidable presence of noise. However, hydrodynamic synchronization of rotators has been investigated only by analytical $[6,11]$ and numerical models [15] or macroscopic experiments [16] that do not take into account noise. A colloidal model for rotators, of the kind used [13] for modeling ciliar beating motions, is still lacking.

In this Letter, we have used two-photon polymerization to build a microscopic model of hydrodynamically coupled propellers driven by radiation pressure. We demonstrate that hydrodynamic interactions alone can synchronize the two rotors, although the applied torques have to be finely tuned. The stationary probability distribution for the phase lag between the two rotors obeys a Fokker-Planck equation for an overdamped particle over a tilted periodic potential [17].

Synchronization between two self-sustained oscillators manifests with the appearance of phase-locked states that survive even when we detune the two oscillators' frequencies within some finite range [3]. Those states are even easily detectable in the presence of noise that would quickly destroy any accidental phase-locking, driving phases away in diffusing random walks. On the other hand, if noise is unbounded, perfect phase-locking never occurs. However, synchronization still will be clearly visible as an intermittent dynamics where rapid phase slips interrupt periods of phase-locking whose lifetime increases 
as we tune the frequencies of the two oscillators $[3,8]$. An ideal micron scale model for studying hydrodynamic synchronization of rotators should provide a precise way of tuning torques, together with some degree of mechanical flexibility. All those features are readily available in the colloidal lightmills introduced by Galajda and Ormos in [18]. These are two-photon polymerized structures measuring a few microns and with a chiral structure similar to that of windmills. Those structures are also provided with an axis that automatically ensures stable trapping and alignment to the axis of an optical trap. Trapping light will be scattered with a nonzero component of orbital angular momentum giving rise to an applied torque. At the same time, trapping light will act as a spring restoring the rotor axis in its equilibrium position and providing the required degree of mechanical flexibility.

The rotors were produced using two-photon excitation photopolymerization by direct laser beam writing from SU8 2007 photoresist as described in detail in [19,20]. We fabricated two types of rotors with mirror symmetry to get clockwise or anticlockwise rotation [Fig. 1(a)]. The optical tweezers were built around a Zeiss Axio Observer A1 inverted microscope with an Olympus UPlanSApo water immersion $60 \mathrm{X} / 1.2$ objective. Two counterspinning rotors were trapped simultaneously by splitting the laser beam (1070 nm, IPG-YLM-10, IPG Photonics) with a spatial light modulator (PLUTO NIR, Holoeye) [21].

By changing the location and power of each trap, we could control independently the rotors' position and applied optical torques. Figure 1(b) shows two rotors spinning in opposite directions at a distance $d$ of $6 \mu \mathrm{m}$ The rotors were spinning at an average frequency of $7.6 \mathrm{~Hz}$. The angular frequency $\Omega$ is given by $\Omega=R M$ where $R$ is the rotational mobility and $M$ is the applied torque. Using the numerical model described later, we can

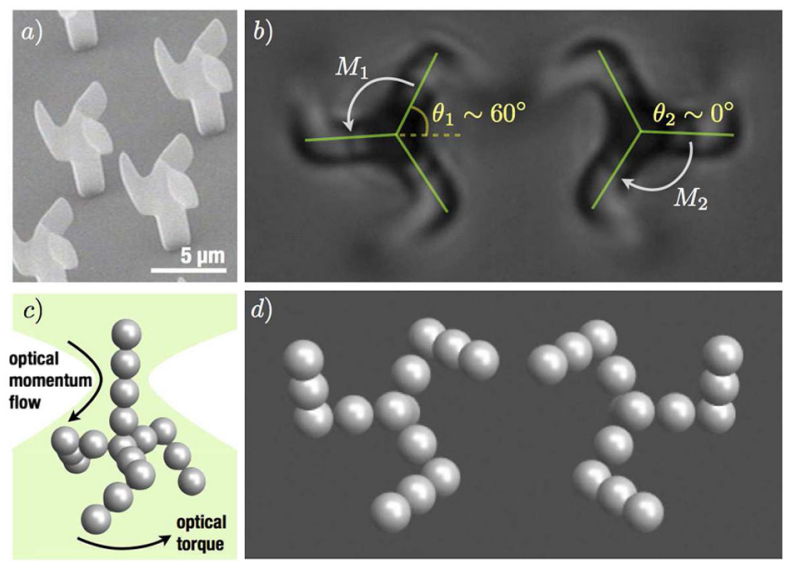

FIG. 1 (color online). (a) SEM images of microrotors over glass substrate. (b) Bright-field image of synchronized microrotors spinning in opposite directions. (c) Rotors are modeled as an array of 17 spherical beads. (d) Snapshot from the numerical simulation. estimate a mobility of about $R=5.3(\mathrm{pN} \mu \mathrm{m} \mathrm{s})^{-1}$, which gives an optical torque of about $9 \mathrm{pN} \mu \mathrm{m}$. Individual rotor phases $\theta_{1}$ and $\theta_{2}$ are defined as in Fig. 1(b) and extracted by a real-time video-tracking software. As a phase difference variable, we choose the quantity $\varphi=\left|\theta_{1}\right|-\left|\theta_{2}\right|$ that gives the appropriate result for rotors spinning in the same or opposite directions. For each distance, we scanned the relative torque by scanning the relative power difference in each trap in steps of order $10^{-3}$. Figure 2(a) shows a data set for the closest investigated distance of $6 \mu \mathrm{m}$. There is a marked tendency for the relative phase to get trapped at values $\varphi \sim 60^{\circ}$ modulo $120^{\circ}$. That corresponds to rotors rotating with arms that tend to stay as close as possible. As we tuned the relative torques toward a better match, the frequency of phase slips was reduced to about one every 20 seconds, that is one every 150 full rotations. It is enough to increase the distance to $7 \mu \mathrm{m}$ to observe a much weaker phase-locking [Fig. 2(b)].

Our numerical approach to the problem consists of describing each rotor as a rigid assembly of $N$ spherical beads with radius $a$ and coordinates $\mathbf{R}_{\alpha}=\left\{\mathbf{r}_{\alpha}^{1}, \ldots, \mathbf{r}_{\alpha}^{N}\right\}$ with $\alpha=1,2$ the rotor index. Each bead will be subject to an unknown force $\mathbf{f}_{\alpha} \mathrm{a}^{j}$, resulting from the internal mechanical interaction with neighboring beads and the external optical force. Hydrodynamic interactions can be
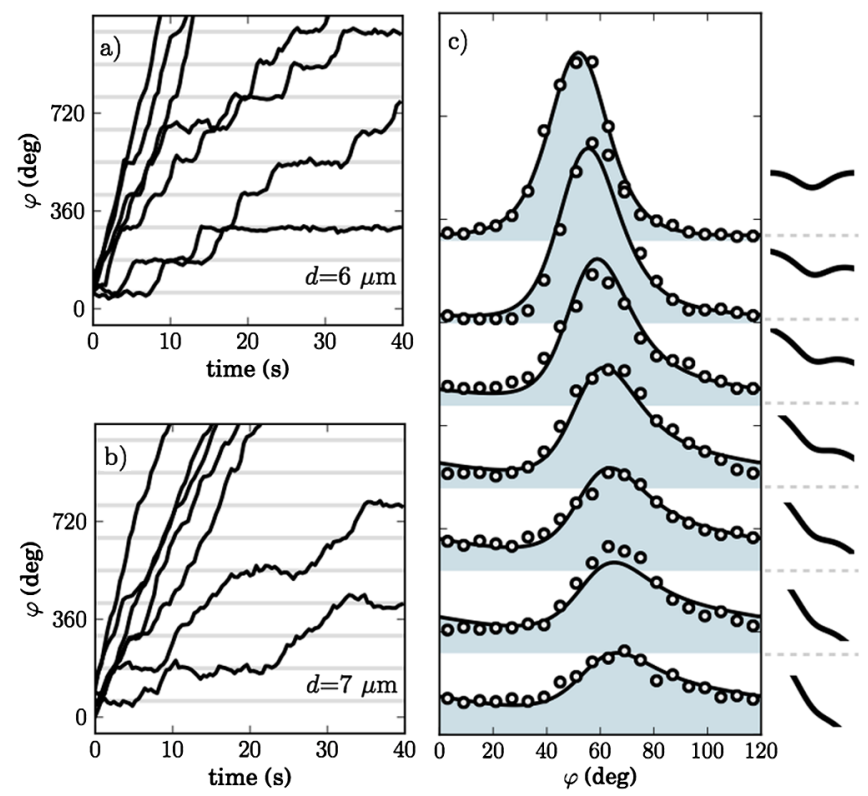

FIG. 2 (color online). Phase lag $\varphi$ dynamics for different values of torque mismatch $\Delta M$. (a) Rotors' distance $d=$ $6 \mu \mathrm{m}$. Each curve corresponds to a given $\Delta M$ that is scanned in steps of order $10^{-3} \mathrm{pN} \mu \mathrm{m}$. Smaller $\Delta M$ correspond to curves with lower average slopes. As $\Delta M$ approaches zero, the phase lag is locked at $60^{\circ}$ modulo $120^{\circ}$ for increasing time intervals. (b) Same plot for a $7 \mu \mathrm{m}$ distance. (c) Open symbols represent the experimental probability distributions for $\varphi$ at $d=6 \mu \mathrm{m}$. Torque difference is increased in the curves from top to bottom. Solid lines are the calculated Fokker-Planck stationary distributions. 
accounted for, at the lowest order, by assuming that each bead is advected by a background flow that is obtained as the sum of the Stokes flows propagating from point forces acting on all the other beads. For rotor 1 this reads

$\dot{\mathbf{r}}_{1}^{j}=m_{0} \mathbf{f}_{1}^{j}+\sum_{k \neq j} \mathbf{G}\left(\mathbf{r}_{1}^{j}-\mathbf{r}_{1}^{k}\right) \cdot \mathbf{f}_{1}^{k}+\sum_{k} \mathbf{G}\left(\mathbf{r}_{1}^{j}-\mathbf{r}_{2}^{k}\right) \cdot \mathbf{f}_{2}^{k}$,

where $m_{0}=(6 \pi \mu a)^{-1}$ is the single bead mobility and $\mathbf{G}(\mathbf{r})=(8 \pi \mu r)^{-1}(1+\hat{\mathbf{r}} \hat{\mathbf{r}})$ is the Oseen tensor. Adding the analogous equation for rotor 2 , we end up with $6 N$ equations for the $12 \mathrm{~N}$ unknown components of the vectors $\dot{\mathbf{r}}_{\alpha}^{j}, \mathbf{f}_{\alpha}^{j}$. We now impose the constraint that each rotor moves as a rigid body and only allow for rotations around the $z$ axis. Calling $\mathbf{r}_{\alpha}^{0}$ the coordinates of a reference point on the $\alpha$ th rotor axis, and $\theta_{\alpha}$ the instantaneous rotor angle around the $z$ axis we have

$$
\dot{\mathbf{r}}_{\alpha}^{j}=\dot{\mathbf{r}}_{\alpha}^{0}+\dot{\theta}_{\alpha} \hat{z} \times\left(\mathbf{r}_{\alpha}^{j}-\mathbf{r}_{\alpha}^{0}\right) .
$$

That amounts to adding another $6 \mathrm{~N}$ equations at the cost of introducing the 4 unknowns $\dot{\mathbf{r}}_{\alpha}^{0}, \dot{\theta}_{\alpha}$. The system of equations can be closed by imposing that the total force acting on each rotor is purely optical and amounts to an elastic restoring force with finite stiffness $k$ plus a constant torque along $z$ given by $M_{\alpha}$ :

$$
\sum_{j=1, N} \mathbf{f}_{\alpha}^{j}=-k \mathbf{r}_{\alpha}^{0}, \quad \sum_{j=1, N}\left(\mathbf{r}_{\alpha}^{j}-\mathbf{r}_{\alpha}^{0}\right) \times \mathbf{f}_{\alpha}^{j}=M_{\alpha} \hat{z} .
$$

For each configuration, we can solve equations (1)-(3) for the speeds $\dot{\mathbf{r}}_{\alpha}^{0}$ and $\dot{\theta}_{\alpha}$ and obtain trajectories for the two coupled rotors. Analytical studies of deterministic minimal models [16,22], made of just one or two spheres per rotor, have shown that in the limit of large separations and small torque differences, it is possible to write an equation of motion for the one cycle average of the phase difference $\bar{\varphi}$ in the form

$$
\dot{\bar{\varphi}}=R\left(-\frac{d U(\bar{\varphi})}{d \bar{\varphi}}+\Delta M\right),
$$

where $\Delta M=\left|M_{1}\right|-\left|M_{2}\right|$ is the applied torque difference, $R$ is the rotational mobility of an isolated structure, and $U$ a periodic function of $\varphi$ that encodes the whole complex structure of hydrodynamic interactions in a term that act as a periodic potential for $\varphi$. A far-field solution of minimal models also shows that $U(\bar{\varphi})=u(\bar{\varphi}) M^{2} / k a^{2}$, where $M$ is the average applied torque modulus and $u$ an adimensional periodic function of $\bar{\varphi}$ whose structure depends solely on geometrical parameters like the shape and relative distance of the rotors. Using numerical simulations of model rotors made of 17 spheres [Fig. 1(c)], we find out that the structure of (4) remains valid even for more complex 3D shapes and shorter separations as in Fig. 1(d). Figure 3 shows the numerically calculated potential $U$ for $k=1 \mathrm{pN} / \mu \mathrm{m}$. Synchronized states correspond to minima in $U$ whose shape mainly depends on $k$ through a multiplicative factor. It is worth noting that, within the approximation order used

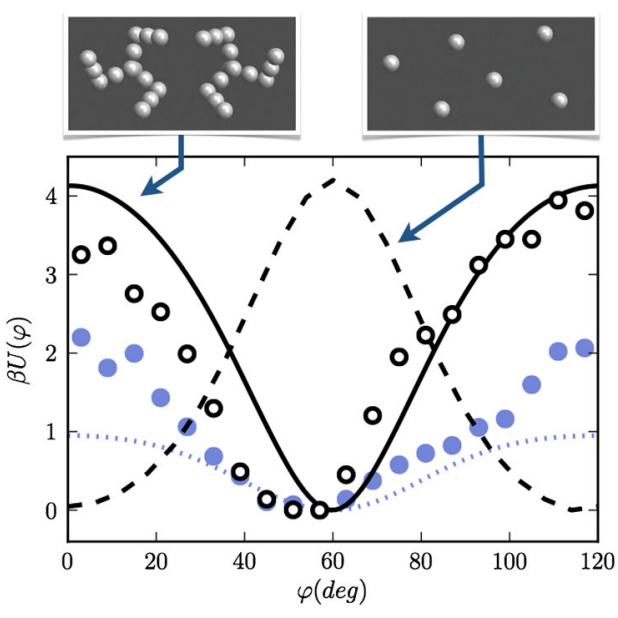

FIG. 3 (color online). Experimental data for the potential $\beta U$ measured at rotor distances $6 \mu \mathrm{m}$ (open circles) and $7 \mu \mathrm{m}$ (filled circles). Solid line and dashed line correspond, respectively, to the 17 and 3 spheres models predictions for $6 \mu \mathrm{m}$ distance and are scaled by an arbitrary factor. Using the same scaling factor, dotted line is the 17 spheres model prediction for $7 \mu \mathrm{m}$ distance.

to derive (4), hydrodynamic interactions do not modulate the mobility term $R$ by which the phase lag $\varphi$ responds to small torque differences. They only appear in the potential term $U$ whose amplitude depends on the average applied torque. It is then reasonable to generalize (4) to a stochastic equation for the instantaneous phase lag $\varphi$ by simply adding to the torque mismatch $\Delta M$ a fluctuating component $\eta$ that, within the present approximation of a constant $R$, plays the role of an additive noise

$$
\dot{\varphi}=R\left(-\frac{d U}{d \varphi}+\Delta M+\eta\right)
$$

The above equation has the form of a stochastic Adler equation $[3,8]$, where $\eta$ has zero average and a white noise spectrum

$$
\langle\eta(t)\rangle=0, \quad\langle\eta(0) \eta(t)\rangle=2 A \delta(t) .
$$

The resulting dynamics will lead to stationary probability distributions that are formally obtained by solving the corresponding Fokker-Planck equation and imposing periodic boundary conditions [17]

$$
P(\varphi)=\frac{1}{Z} e^{-\beta U(\varphi)} \int_{\varphi}^{\varphi+2 \pi / 3} e^{\beta\left[U\left(\varphi^{\prime}\right)-\Delta M\left(\varphi^{\prime}-\varphi\right)\right]} d \varphi^{\prime},
$$

where $\beta=1 / R A$ and $Z$ is a normalization factor that can be determined by imposing $\int_{0}^{2 \pi / 3} P(\varphi) d \varphi=1$. The case of equal torque corresponds to Brownian motion over a periodic potential so that the stationary distribution (7) reduces to the Boltzmann equilibrium distribution

$$
P(\varphi) \propto e^{-\beta U(\varphi)} .
$$

That suggests a straightforward procedure to extract the potential from the experimental data. If we identify the 
equal torque condition as that leading to the most pronounced peak in the probability distribution $P(\varphi)$, then we can obtain the potential as $\beta U(\varphi)=$ $-\log [P(\varphi)]$. If we apply that procedure using, as the equal torque case, the uppermost histogram in [Fig. 2(c)], we end up with the potential reported in Fig. 3. A maximum in the probability distribution corresponds to a minimum at about $60^{\circ}$ in the potential $U$. It is worth noting that, if we model our rotors with a minimal three spheres system, of the kind investigated in [16], we always end up with a minimum at $0^{\circ}$ that is with rotor arms belonging to different rotors always trying to maximize their distance (see dashed line in Fig. 3). However, in both numerical models, the dissipation shows a maximum at $0^{\circ}$ and a minimum at $60^{\circ}$. This observation confirms the existence of a link between synchronized states and extrema in the dissipation function $[5,15]$. Whether synchronization corresponds to minimum or maximum dissipation can depend critically on the detailed shape of the rotors. From Fig. 3 we extract a potential barrier of about 3 in units of $\beta^{-1}$ with a maximum slope, or maximum "synchronizing" torque, of about $M_{c}=$ $6 \beta^{-1}$. If we now assume that all noise comes from thermal agitation, we would expect $\beta^{-1}$ to be of order $k_{B} T=4 \times 10^{-3} \mathrm{pN} \mu \mathrm{m}$. Therefore, no stable synchronized state would exist for torque mismatches larger than $M_{c} \sim 2 \times 10^{-2} \mathrm{pN} \mu \mathrm{m}$ that is only $1 / 1000$ of the applied torque. That is also consistent with the need for performing very fine power scans to observe synchronization. A similar sensitivity of synchronization from frequency mismatch was also observed in coupled oscillating colloids [13], although synchronization is much more robust in that case probably due to the time asymmetric character of the beads' motions. When slightly increasing the rotors' distance from 6 to $7 \mu \mathrm{m}$, a marked decrease of about a factor of two is found in the experimental potential $U$ (Fig. 3). The numerical prediction is slighty lower (dotted line in Fig. 3) and initially falls off faster than the three spheres' model prediction $\sim 1 / r^{7}$. When bringing the simulated rotors at distances larger than about $11 \mu \mathrm{m}$, stable synchronized positions switch to $0^{\circ}$ as in the three spheres' case. This fact suggests that observed synchronization at $60^{\circ}$ could be due to higher order terms in the large-distance expansion of flow fields. Unfortunately, synchronization cannot be detected at $11 \mu \mathrm{m}$, where the numerical model predicts a reduction in $U$ by more than 1000 times with respect to the closest distance investigated $(6 \mu \mathrm{m})$.

We can now use the obtained potential in Fig. 3 and derive the stationary distributions corresponding to unbalanced torques from (7). Although we don't know the exact value of the applied torques, we scanned trap powers in uniform steps, so that the torque differences will be evenly spaced $\Delta M=\Delta M_{0} i$, with $i$ a progressive integer. We are then left with the only free parameter $\alpha=\beta \Delta M_{0}$. By a fitting procedure, we obtain $\alpha=2$ which allow us to produce through (7) all other probability distributions as shown in Fig. 2(c). This provides a strict quantitative test of the phenomenological equation (5), which contains a number of conjectures, such as the statistical properties (6) of fluctuating torque $\eta$. Knowing $\alpha$, we can now reconstruct the full tilted potentials $U-R \Delta M \varphi$ which are reported in the right column of Fig. 2(c). For the highest torque mismatches, the tilted potentials no longer have a stable equilibrium position, although a peak is still visible in the corresponding $P(\varphi)$. This is due to the fact that, even if the phase lag always drifts without getting locked in local minima, it slows down, spending more time in the neighborhood of $60^{\circ}$, where the slope of the tilted potential is minimum.

In conclusion, we have demonstrated that two mesoscopic rotors can be synchronized by hydrodynamic interactions alone. However, the strength of the coupling mechanism is so weak that relative torques have to be tuned with a resolution of $10^{-3}$. Dealing with a mesoscopic model, we have studied synchronization as a stochastic process and shown that the phase lag is distributed according to the steady solution of a Fokker-Planck equation. As a final remark, the presence of noise and the natural dispersity that occurs in biological motors and propeller shapes will make hydrodynamic synchronization of natural rotators, like flagellar bundles on nearby bacteria, an extremely weak phenomenon when compared to collective phenomena in cilia that beat with time-irreversible patterns.

R.D.L. acknowledges funding from IIT-SEED BACTMOBIL Project and MIUR-FIRB Project No. RBFR08WDBE. Participants from Szeged acknowledge Grants OTKA K No. 84335 and TÁMOP No. 4.2.2/B10/1-2010-0012.

*roberto.dileonardo@phys.uniroma1.it

[1] A. K. Jain, Phys. Rep. 109, 309 (1984).

[2] R. F. Schmidt and G. Thews, Human Physiology (Springer, New York, 1983).

[3] A. Pikovsky, M. Rosenblum, and J. Kurths, Synchronization, A Universal Concept in Nonlinear Sciences (Cambridge University Press, New York, 2001).

[4] J. Happel and H. Brenner, Low Reynolds Number Hydrodynamics (Kluwer Academic, Dordrecht, 1983).

[5] G. J. Elfring and E. Lauga, Phys. Rev. Lett. 103, 088101 (2009).

[6] N. Uchida and R. Golestanian, Phys. Rev. Lett. 106, 058104 (2011).

[7] M. Polin, I. Tuval, K. Drescher, J. P. Gollub, and R. E. Goldstein, and Science 325, 487 (2009).

[8] R. E. Goldstein, M. Polin, and I. Tuval, Phys. Rev. Lett. 103, 168103 (2009).

[9] R. E. Goldstein, M. Polin, and I. Tuval, Phys. Rev. Lett. 107, 148103 (2011).

[10] M. C. Lagomarsino, P. Jona, and B. Bassetti, Phys. Rev. E 68, 021908 (2003).

[11] P. Lenz and A. Ryskin, Phys. Biol. 3, 285 (2006). 
[12] A. Vilfan and F. Jülicher, Phys. Rev. Lett. 96, 058102 (2006).

[13] J. Kotar, M. Leoni, B. Bassetti, M. C. Lagomarsino, and P. Cicuta, Proc. Natl. Acad. Sci. U.S.A. 107, 7669 (2010).

[14] R. Golestanian, J. M. Yeomans, and N. Uchida, Soft Matter 7, 3074 (2011).

[15] M. Reichert and H. Stark, Eur. Phys. J. E 17, 493 (2005).

[16] B. Qian, H. Jiang, D. A. Gagnon, K. S. Breuer, and T. R. Powers, Phys. Rev. E 80, 061919 (2009).
[17] H. Risken, The Fokker-Planck Equation (Springer-Verlag, Berlin, 1989).

[18] P. Galajda and P. Ormos, Appl. Phys. Lett. 78, 249 (2001).

[19] L. Kelemen, S. Valkai, and P. Ormos, Appl. Opt. 45, 2777 (2006).

[20] L. Kelemen, P. Ormos, and G. Vizsnyiczai, J. Eur. Opt. Soc. 6, 1029 (2011).

[21] M. Reicherter, T. Haist, E. Wagemann, and H. Tiziani, Opt. Lett. 24, 608 (1999).

[22] T. Niedermayer, B. Eckhardt, and P. Lenz, Chaos 18, 037128 (2008). 